\title{
Use of Functional Balance Measurement Tools to Predict the Fear of Falling in Faller and Non-Faller Chronic Stroke Patients
}

\author{
Shamsi Jamali', Akram Azad ${ }^{1}$, Ghorban Taghizadeh ${ }^{1,2 *}$ \\ 1. Department of Occupational Therapy, School of Rehabilitation Sciences, Iran University of Medical Sciences, Tehran, \\ Iran \\ 2. Rehabilitation Research Center, School of Rehabilitation Sciences, Iran University of Medical Sciences, Tehran, Iran
}

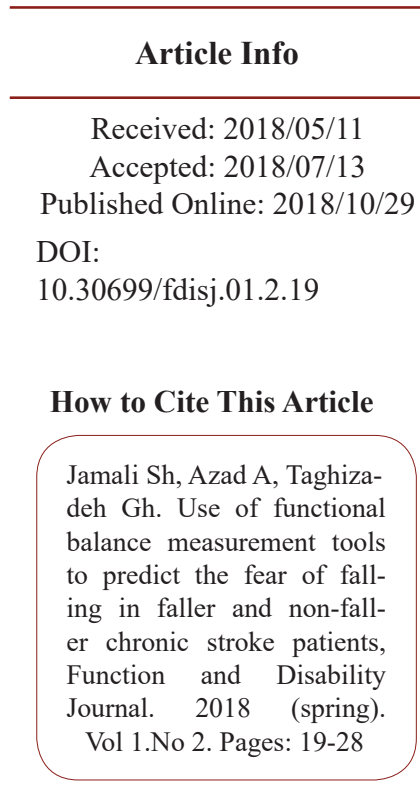

Use your device to scan and read the article online

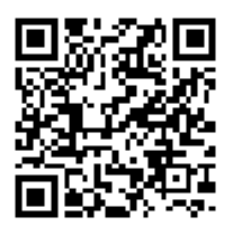

\begin{abstract}
Background and Objective: Fear of falling is a major problem among people suffering from stroke. This study aimed to investigate the relationship between functional balance (FB) and fear of falling (FOF) in chronic stroke patients with and without history of falling and to identify the best measurement tool for FB which can assess the FOF in these patients.
\end{abstract}

Methods: Eighty-two participants with chronic stroke with the mean age of 54.90 $( \pm 11.30)$ years participated in this non-experimental cross sectional study. They were divided into faller and non-faller groups based on their history of falling during the last month. The FB was measured by Berg Balance Scale (BBS), Dynamic Gait Index (DGI), Tinetti Balance Test, and Functional Reach Test (FRT). The Persian versions of the self-completed Fall Efficacy Scale-International (FES-I) and Activity-specific Balance-Confidence scale (ABC) were used to evaluate FOF.

Results: The results of this study showed that the correlation between FES-I with FRT, BBS, DGI and Tinetti Balance Test were $-0.38,-0.67,-0.44$ and -0.62 , in faller group and $-0.11,-0.59,-0.31$ and -0.24 in non-faller group. The correlation between ABC and FRT, BBS, DGI and Tinetti Balance Test were 0.58, 0.48, 0.68 and 0.57 in faller group and $0.17,0.55,0.45$ and 0.44 in non-faller group. The results of multiple regression analysis showed that BBS was the best predictor for FOF (which explained $25 \%$ and $31 \%$ of total variance of FES-I and ABC in faller group as well as $68 \%$ and $42 \%$ of total variance of FES-I and ABC in non-faller group).

Conclusion: The results of this study showed a low to moderate correlation between FB and FOF in both groups of faller and non-faller chronic stroke patients. Furthermore, BBS seems to be the best functional balance measurement to predict FOF in these patients.

Keywords: Chronic stroke; Fear of falling; Functional balance

\section{Introduction}

Stroke is the fifth most common cause of neurologic dysfunction in adults (Mozaffarian et al., 2015) which results in impaired postural and balance control as well as bilateral coordination, weakness of limbs and trunk control, and limitations in activities of daily living (ADLs) (Bensoussan et al., 2007).

Balance impairment is one of the most common motor problems following stroke which affects about $83 \%$ of patients with stroke and increases the risk and the fear of falling (FOF) in some patients (Monfared, Mehraban, \& Boroumand, 2015). The FOF is defined as a concern of falling during ADLs performance (Shaikh \& asghar Hosseini, 2016). According to the previous studies, of all 33 stroke patients, 29 subjects experienced the FOF within 27-28 months after falling 
(Monfared et al., 2015). Patients with stroke are more than twice as likely to be at risk of falling as elderly. The FOF was seen in approximately $88 \%$ of stroke patients with a history of falling. The stroke patients with FOF avoid different activities which may lead to restricted mobility, reduced functional capabilities, and loss of functional independence. It is noteworthy that this avoidance leads to the muscle atrophy and weakens the balance performance which finally increases the risk of falling and FOF (Schinkel-Ivy, Inness, \& Mansfield, 2016). Various studies among stroke population have shown that the severity of disease, the balance impairment, and the FOF may be related to the lower physical activity levels (Goh, Nadarajah, Hamzah, Varadan, \& Tan, 2016; Rosén, Sunnerhagen, \& Kreuter, 2005; Shaikh \& asghar Hosseini, 2016). Understanding the relationship between FOF and balance is essential for better evaluation of patients as well as designing more effective clinical interventions and rehabilitation programs.

There are different tools available to measure functional balance (FB) and the FOF in medical and research environments. Using a variety of such tools in each field can be time consuming. Hence, finding a tool which is capable of assessing both areas can be very helpful. Therefore, the purpose of this study was to investigate the relationship between FB measures and the FOF and to identify the balance measurement tools capable of predicting the FOF in chronic stroke patients with and without a history of falling.

\section{Materials and Methods}

\section{Participants}

In this cross-sectional study, 82 subjects with chronic stroke were recruited by simple non-probability method from neurologic outpatient clinics in Tehran. They were divided into faller and non-faller groups based on their history of falling during the last month (Afridi, Ayesha et al. 2015). The sample size was determined based on the results of a pilot study. Individuals were included to the study if they: had chronic stroke (i.e. more than 12 months since stroke) diagnosed by a neurologist; obtained a score greater than 21 on the Mini Mental Status Examination (Godefroy et al., 2011) and a score between 0 to 3 on the Modified Ranking Scale (MRS); were able to understand instructions of tests in Persian; had no other neurologic or orthopedic disorders other than stroke; and did not use medications that affect their balance. The participants were excluded if they did not cooperate in the implementation of the tests.

The patients signed the informed consent form before participating in this study and could leave the process whenever they wished. The study was approved by the Ethics Committee of Iran University of Medical Sciences (IR.IUMS.res. 1395. 9411355001).

\section{Procedures}

The participants completed the demographic data questionnaire including age, sex, dominant hand, affected side, time passed from stroke, history of falling and the number of fallings during the last month. The severity of stroke was determined according to the Modified Ranking Scale (MRS). The FB was measured by Berg Balance Scale (BBS), Dynamic Gait Index (DGI), Tinetti Balance Test and Functional Reach Test (FRT). The FOF was determined by Fall Efficacy Scale-International (FES-I) and Activitiesspecific Balance Confidence (ABC) questionnaires. The tests were conducted by an experienced occupational therapist in a randomized order and in a room with stable conditions. All the tests were performed for each participant in one session and the time duration of evaluations varied between 30-60 minutes per patient.

\section{Instruments}

\section{Modified Ranking Scale}

The MRS scale was used to assess the general disability and stroke complications and to predict poststroke functional improvement. The scores of this scale range from 0 to 5 ; zero implies no symptoms while grade 5 indicates severe damage (Mehdizadeh, Mehraban, \& Zahediyannasab, 2017). The MRS has good validity in stroke patient (Cincura et al., 2009; Ghandehari et al., 2012). Chronic stroke patients who scored 0 to 3 according to the MRS were entered in this study. 


\section{Berg Balance Scale (BBS)}

BBS, a basic functional balance test, consists of 14 items, each of which is scored from 0 to 4 . The total score of the BBS is 56 which indicates the excellent balance, while the score 0 indicates severe balance impairment. The Persian version of BBS has been shown to be a reliable tool for the elderly and Multiple Sclerosis (ICC $=0.93, \mathrm{ICC}=0.99$; respectively) (Azad, Taghizadeh, \& Khaneghini, 2011; Salavati et al., 2012).

\section{Tinetti Balance Test}

It is a valid test for gait and balance ability. This test consists of 9 items for balance and 7 items for gait assessment. Each item is scored in the range of 0 (very weak performance) to 2 (acceptable performance). The maximum total score is 28 . Good to excellent inter-rater and intra-rater reliability of this test has been reported in stroke patients (ICC $=0.84)$ (Canbek, Fulk, Nof, \& Echternach, 2013; Tinetti, Williams, \& Mayewski, 1986).

\section{Dynamic Gait Index (DGI)}

This test is used to assess and diagnose falling and includes 8 items. Each item is scored on a 4-point ordinal scale, within which the 0 represents severe impairment and 3 represents normal function. The range of total score is 0 to 24 points. The higher scores indicate better ability to move normally. This test has an excellent reliability for assessing chronic stroke patients $(\mathrm{ICC}=0.96)$ (Abdiani, Golpayegani, \& Khajavi, 2014; Eghlidi et al., 2017; Jonsdottir \& Cattaneo, 2007).

\section{Activities-specific Balance Confidence scale (ABC)}

This scale evaluates the self-efficacy and confidence in balance in wide range of daily activities. This scale consists of 16 self-report items which a patient scores them according to his/her sense of confidence in balance. The total score ranges from 0 to 100 . The Persian version of this scale has been shown to be a reliable scale among stroke patients (Azad, Taghizadeh, Mohammadian, Mohammadinezhad, \& Lajevardi, 2017).

\section{Fall Efficacy Scale-International (FES-I)}

FES-I is a 16-item scale which measures the FOF during 10 daily activities. The range of total scores can vary from 16 to 64 . The higher score indicates higher FOF. The Persian version of the FES-I has a high validity and reliability within the stroke population (Azad, Mehraban, Mehrpour, \& Mohammadi, 2014; Mehdizadeh et al., 2016).

\section{Functional Reach Test (FRT)}

It is a quick and single-task dynamic test, in which the participant is asked to reach forward as far as possible without taking a step or touching the wall. The distance (based on inch or $\mathrm{cm}$ ) between the start and the end point is then measured using the head of the metacarpal of the third finger as the reference point. The validity and reliability of the FRT have been established (inter-rater: ICC $=0.98$ and intrarater: $\mathrm{ICC}=0.92$ ) (Demura \& Yamada, 2007; Smith, Hembree, \& Thompson, 2004).

\section{Statistical analysis}

The normal distributions of data were evaluated using Shapiro-Wilks test. The Pearson correlation coefficient test was used to examine the correlation between FOF and FB measures in faller and non-faller groups (Daneshjoo, Azad, Mandehgary, Mehdizadeh, \& Taghizadeh, 2016). The strength of the correlation was determined based on the Munro's descriptive scale as follows: $r$ values of $0.00-0.25,0.26-0.49$, 0.50-0.69, 0.70-0.89 and 0.90-1.00 indicates little, low, moderate, high and very high correlations, respectively (Domholt , 2005). A multiple regression analysis was performed to identify the best predictor tool for the FOF.

\section{Results}

\section{Participant's characteristics}

Eighty-two chronic stroke patients (60 males and 22 females) by the mean age of $54.90 \pm 11.30$ years were participated in this study. Thirty-two participants $(38.8 \%)$ reported a history of falling in the last month while fifty participants $(60.2 \%)$ did not report any falling history during the same time. The descriptive data of the variables is reported in Table 2. 
Table 1. Demography characteristics of patients with stroke $(n=82)$

\begin{tabular}{|c|c|c|c|}
\hline Variables & \multicolumn{2}{|c|}{ Mean $( \pm$ SD) } & Min-Max \\
\hline Age (year) & \multicolumn{2}{|c|}{$54.90( \pm 11.30)$} & $27-76$ \\
\hline Time since diagnosis (year) & \multicolumn{2}{|c|}{$6.08( \pm 5.76)$} & $2-10$ \\
\hline Mini Mental Status Examination (score) & \multicolumn{2}{|c|}{$25.90( \pm 2.75)$} & $21-30$ \\
\hline Variables & \multicolumn{2}{|c|}{ Frequency (\%) } & \\
\hline male & \multicolumn{2}{|c|}{$60(72.3 \%)$} & \\
\hline \multirow{2}{*}{ Sex } & female & $22(26.5 \%)$ & \\
\hline & right & $74(89.2 \%)$ & \\
\hline \multirow{2}{*}{ Dominant hand } & Left & $8(9.6 \%)$ & \\
\hline & right & $48(38.6 \%)$ & \\
\hline \multirow{2}{*}{ Affected side } & left & $34(41.0 \%)$ & \\
\hline & yes & $32(38.6 \%)$ & \\
\hline \multirow{2}{*}{$\begin{array}{l}\text { History of Falling } \\
\text { (past one-month ) }\end{array}$} & No & $50(60.8 \%)$ & \\
\hline & One & $30(36.1 \%)$ & \\
\hline Numbers of falling & Two & $2(2.4)$ & \\
\hline
\end{tabular}

Table 2. Characteristics of FOF and FB measurements

\begin{tabular}{cccc} 
Measures & Tools & Min-Max & Mean ( \pm SD) \\
\hline & FRT & $12-42$ & $25.4( \pm 5.96)$ \\
FB & BBS & $26-56$ & $45.67( \pm 7.19)$ \\
\hline & DGI & $8-24$ & $21.54( \pm 3.94)$ \\
\hline FOF & Tinetti & $10-28$ & $26.19( \pm 7.5)$ \\
\hline
\end{tabular}

Abbreviations: FOF: Fear of Falling; FB: Functional Balance

The relationship between $\mathrm{FB}$ and FOF measures in chronic stroke patients with a history of falling

There was a significant low and negative relationship between the total score of the FES-I and FRT $(r=-0.38)$ as well as DGI $(r=-0.44)$ in the faller group. The results also showed a significant moderate and negative relationship between the total score of the FES-I with BBS $(r=-0.67)$ and Tinetti Balance Test $(r=-0.62)$ in this group. There was a significant moderate and positive relationship between the total score of the ABC with FRT $(r=0.58)$, DGI $(r=0.68)$ and Tinetti Balance Test $(r=0.57)$ in the faller group. Moreover, a significant low and positive relationship was found between the total score of the $\mathrm{ABC}$ and BBS $(r=0.48)$ in this group (Table 3$)$.

Fifty-two percent of the variance of the FES-I in faller group was explained by FRT, BBS, DGI and Tinetti Balance Test. Fifty-four percent of the variance of the ABC test in this group was explained by FRT, BBS, DGI and Tinetti tests (Table 4). 
Table 3. Correlation (r, P-value) between FOF and FB in stroke patients with and without a history of falling $(n=82)$

\begin{tabular}{|c|c|c|c|c|c|}
\hline History of falling & Tools & FRT & BBS & DGI & Tinetti \\
\hline \multirow{2}{*}{ Faller } & FES-I & $\begin{array}{c}-0.38 \\
*(0.03)\end{array}$ & $\begin{array}{c}-0.67 \\
*(0.000)\end{array}$ & $\begin{array}{c}-0.44 \\
*(0.01)\end{array}$ & $\begin{array}{c}-0.62 \\
*(0.000)\end{array}$ \\
\hline & $\mathrm{ABC}$ & $\begin{array}{c}0.58 \\
*(0.001)\end{array}$ & $\begin{array}{c}0.48 \\
*(0.005)\end{array}$ & $\begin{array}{c}0.68 \\
*(0.000)\end{array}$ & $\begin{array}{c}0.57 \\
*(0.001)\end{array}$ \\
\hline \multirow{2}{*}{ Non-faller } & FES-I & $\begin{array}{l}-0.11 \\
(0.43)\end{array}$ & $\begin{array}{c}-0.59 \\
*(0.000)\end{array}$ & $\begin{array}{c}-0.31 \\
*(0.026)\end{array}$ & $\begin{array}{c}-0.24 \\
(0.093)\end{array}$ \\
\hline & $\mathrm{ABC}$ & $\begin{array}{c}0.17 \\
(0.24)\end{array}$ & $\begin{array}{c}0.55 \\
*(0.000)\end{array}$ & $\begin{array}{c}0.45 \\
*(0.001)\end{array}$ & $\begin{array}{c}0.44 \\
*(0.001)\end{array}$ \\
\hline
\end{tabular}

Abbreviations: FES-I: Fall Efficacy Scale-International; ABC: Activities-specific Balance Confidence; FRT: Functional Reach Test; BBS: Berg Balance Scale; DGI: Dynamic Gait Index; Tinetti: Tinetti Balance and Gait Assessment; FOF: Fear Of Falling; FB: Functional Balance; $\left({ }^{*} P \leq 0.05\right)$

Table 4. A summary of simultaneous multiple regression analysis, FOF measures as the dependent variable and FB measures as the independent variable in patient with stroke $(n=82)$

\begin{tabular}{|c|c|c|c|c|c|c|c|}
\hline $\begin{array}{l}\text { History of } \\
\text { falling }\end{array}$ & $\begin{array}{c}\text { FOF } \\
\text { measures }\end{array}$ & $\begin{array}{c}\text { FB } \\
\text { measures }\end{array}$ & $\mathbf{R}$ & $\mathbf{R}^{2}$ & $\underset{\mathbf{R}^{2}}{\text { Adjusted }}$ & Beta coefficient & $\begin{array}{c}P \text {-value for } \\
\text { Beta coefficient }\end{array}$ \\
\hline \multirow{8}{*}{ Faller } & \multirow{4}{*}{ FES-I } & FRT & \multirow{4}{*}{0.72} & \multirow{4}{*}{0.52} & \multirow{4}{*}{0.45} & -0.65 & $* 0.030$ \\
\hline & & BBS & & & & -0.25 & 0.120 \\
\hline & & DGI & & & & -0.23 & 0.471 \\
\hline & & Tinetti & & & & 0.36 & 0.151 \\
\hline & \multirow{4}{*}{$\mathrm{ABC}$} & FRT & \multirow{4}{*}{0.74} & \multirow{4}{*}{0.54} & \multirow{4}{*}{0.48} & -0.15 & 0.597 \\
\hline & & BBS & & & & 0.31 & $* 0.049$ \\
\hline & & DGI & & & & 0.18 & 0.566 \\
\hline & & Tinetti & & & & 0.50 & 0.047 \\
\hline \multirow{5}{*}{ Non-faller } & \multirow{2}{*}{ FES-I } & BBS & \multirow{2}{*}{0.60} & \multirow{2}{*}{0.36} & \multirow{2}{*}{0.33} & -0.68 & $* 0.000$ \\
\hline & & DGI & & & & 0.13 & 0.393 \\
\hline & \multirow{3}{*}{$\mathrm{ABC}$} & BBS & \multirow{3}{*}{0.57} & \multirow{3}{*}{0.32} & \multirow{3}{*}{0.28} & 0.42 & $* 0.014$ \\
\hline & & DGI & & & & 0.06 & 0.770 \\
\hline & & Tinetti & & & & 0.15 & 0.425 \\
\hline
\end{tabular}

Abbreviations: FES-I: Fall Efficacy Scale-International; ABC: Activities-specific Balance Confidence; FRT: Functional Reach Test; BBS: Berg Balance Scale; DGI: Dynamic Gait Index; Tinetti: Tinetti Balance and Gait Assessment; FOF: Fear of Falling; FB: Functional Balance (*P $\leq 0.05)$

The relationship between FB and FOF measures in chronic stroke patients without a history of falling

No significant relationship was found between the total score of the FES-I and FRT in the non-faller group $(P>0.05)$. There was a significant moderate and positive relationship between the total score of the FES-I and BBS $(r=-0.59)$ in this group. In addition, a significant low and negative relationship was observed between the total score of the FES-I and DGI $(r=-0.31)$ as well as Tinetti Balance Test $(r=-0.24)$ in chronic stroke patients without a history of falling. However, no significant relationship was found between the total score of the ABC and FRT $(P>0.05)$. There was a significant moderate and positive relationship between the total score of the $\mathrm{ABC}$ and $\mathrm{BBS}$ $(r=0.55)$ in the non-faller group. A significant low and positive relationship was found between the total score of the ABC and DGI $(r=0.45)$ as well as Tinetti Balance Test $(r=0.44)$ in this group (Table 3$)$. 
Thirty-six percent of the variance of the FES-I in non-faller participants was explained by BBS test (Table 4).

\section{Discussion}

This study was the first one to examine the relationship between functional balance and FOF measures in two groups of stroke patients with and without history of falling. Another goal of the study was to determine the best FB measure to predict the FOF.

The results of this study showed that there was low to moderate correlation between FB measures and FOF in patients with and without a history of falling. Moreover, there was no significant relationship between FRT and Tinetti balance scores with the FOF in patients without a history of falling. This may be the reason that FRT and Tinetti Balance Test evaluate the FB using a number of tasks, while the latter tools are in the form of subjective reports asking about the anxieties that patients have about falling which may not be a concern in patients who have not experienced any falling.

The results of multiple regression analysis showed that FRT and BBS had the most effect on FOF in both groups of fallers and non-fallers $(65 \%$ and $68 \%$ of total variance, respectively).

Furthermore, there was a moderate association between the scores of FB measures and the FOF in both groups. It is notable that there was no significant correlation between the FRT score and the total score of the $\mathrm{ABC}$ in patients without a history of falling which may be because the FRT test evaluates exclusively one aspect of the balance.

The results of the multiple regression analysis for FOF variables which were related to the FB, showed that BBS had the highest effect in subjects with and without a history of falling (31\% and $42 \%$; respectively) while the score of Tinetti Balance Test had effect on the FOF only in faller patients $(50 \%$ of total variance). The previous studies have shown that patients with chronic stroke tend to be more concerned about falling as compared to the healthy individuals of the same age regardless of their history of falling. This fear is associated with the low functional mo- bility (Cho, Yu, \& Rhee, 2015; Rosén et al., 2005; Shaikh \& asghar Hosseini, 2016).

Schmid study showed that chronic stroke patients with fear of falling had a significantly lower performance than those with no history of falling. Although in some studies, there was no significant difference between the total score of BBS in individuals with and without fear of falling in the acute phase of stroke, the Schmid study showed that individuals with chronic stroke without FOF had a higher BBS score than the ones with FOF (Schmid et al., 2015). This finding indicates that BBS can be a valid tool to predict the confidence balance associated with the fear of falling in patients with chronic stroke.

Since the participants of this study were in the chronic phase following the stroke, they may adapt to their disabilities and learn to avoid the activities which results in falling and the postures which make it difficult to maintain the balance (Belgen, Beninato, Sullivan, \& Narielwalla, 2006). Thus, it would have been better to also include those with a stroke occurrence within 6 months to a year.

One of the limitations of this study was the low sample size. Moreover, the majority of the participants had a high performance. These limitations may limit the generalizability of the current study results.

\section{Conclusion}

This study showed that there is a low to moderate relationship between the FOF and the FB measured by BBS, DGI, Tinetti Balance Test and FRT. The BBS would be the best predictor of FOF in patients with chronic stroke.

\section{Acknowledgement}

This work was supported by Rehabilitation Research Center, School of Rehabilitation Sciences, Iran University of Medical Sciences, Tehran, Iran [Grant Number: 96-02-125-31212].

\section{Conflict of interest statement}

Authors declared no conflict of interest. 


\section{References}

Abdiani, M., Golpayegani, M., \& Khajavi, D. (2014). Validity and reliability of Persian version of dynamic gait index in older men. Iranian Journal of Ageing, 9(2), 124133.

Afridi, A., Maqbool, S., \& Malik, A. N. (2015). Relationship between fear, fall \& balance in cummunity dwelling older adults. Pakistan Journal of Neurological Sciences (PJNS), 10(2), 5-8.

Azad, A., Mehraban, A. H., Mehrpour, M., \& Mohammadi, B. (2014). Clinical assessment of fear of falling after stroke: valid-ity, reliability and responsiveness of the Persian version of the Fall Efficacy Scale-International. Medical journal of the Islamic Republic of Iran, 28, 131.

Azad, A., Taghizadeh, G., \& Khaneghini, A. (2011). Assessments of the reliability of the Iranian version of the Berg Balance Scale in patients with multiple sclerosis. Acta Neurol Taiwan, 20(1), 22-28.

Azad, A., Taghizadeh, G., Mohammadian, E., Mohammadinezhad, T., \& Lajevardi, L. (2017). Persian Translation and Test-retest Reliability of the Activities-specific Balance Confidence Scale in Iranian Chronic Stroke. Journal of Modern Rehabilita-tion, 10(2), 74-79.

Belgen, B., Beninato, M., Sullivan, P. E., \& Narielwalla, K. (2006). The association of balance capacity and falls self-efficacy with history of falling in communitydwelling people with chronic stroke. Archives of physical medicine and rehabilitation, 87(4), 554-561.

Bensoussan, L., Viton, J.-M., Schieppati, M., Collado, H., de Bovis, V. M., Mesure, S., \& Delarque, A. (2007). Changes in pos-tural control in hemiplegic patients after stroke performing a dual task. Archives of physical medicine and rehabilitation, 88(8), 1009-1015.

Canbek, J., Fulk, G., Nof, L., \& Echternach, J. (2013). Test-retest reliability and construct validity of the tinetti performance-oriented mobility assessment in people with stroke. Journal of Neurologic Physical Therapy, 37(1), 14-19.

Cho, K., Yu, J., \& Rhee, H. (2015). Risk factors related to falling in stroke patients: a cross-sectional study. Journal of physical therapy science, 27(6), 1751-1753.

Cincura, C., Pontes-Neto, O. M., Neville, I. S., Mendes,
H. F., Menezes, D. F., Mariano, D. C., De Queiroz, D. C. (2009). Valida-tion of the National Institutes of Health Stroke Scale, modified Rankin Scale and Barthel Index in Brazil: the role of cultural adaptation and structured interviewing. Cerebrovascular Diseases, 27(2), 119-122.

Daneshjoo, F., Azad, A., Mandehgary, M., Mehdizadeh, M., \& Taghizadeh, G. (2016). Correlation between lower and higher order sensory functions and manual dexterity in dominant and non-dominant hand of patients with idiopathic Parkinson's dis-ease. Journal of Basic and Clinical Pathophysiology, 4(2), 27-36.

Demura, S.-i., \& Yamada, T. (2007). Simple and easy assessment of falling risk in the elderly by functional reach test using elastic stick. The Tohoku journal of experimental medicine, 213(2), 105-111.

Domholt, E. (2005). Rehabilitation research: Principles and applications WB Saunders. Philadelphia.

Eghlidi, J., Kalantari, M., Soltanpour, H., Akbar-Fahimi, M., Akbarzadeh, A., BELADI, M. N., \& Baghori, D. (2017). Validity of the Persian Version of Dynamic Gait Index (DGI-P) in Individuals with Multiple Sclerosis.

Ghandehari, K., Ghandehari, K., Saffarian-Toosi, G., Masoudinezhad, S., Yazdani, S., Nooraddin, A., Abrishamchi, F. (2012). Comparative interrater reliability of Asian Stroke Disability Scale, modified Rankin Scale and Barthel Index in patients with brain infarction. ARYA atherosclerosis, 8(3), 153.

Godefroy, O., Fickl, A., Roussel, M., Auribault, C., Bugnicourt, J. M., Lamy, C., Petitnicolas, G. (2011). Is the montreal cogni-tive assessment superior to the minimental state examination to detect poststroke cognitive impairment?: a study with neuro-psychological evaluation. Stroke, 42(6), 1712-1716.

Goh, H.-T., Nadarajah, M., Hamzah, N. B., Varadan, P., \& Tan, M. P. (2016). Falls and fear of falling after stroke: a case-control study. PM\&R, 8(12), 1173-1180.

Jonsdottir, J., \& Cattaneo, D. (2007). Reliability and validity of the dynamic gait index in persons with chronic stroke. Ar-chives of physical medicine and rehabilitation, 88(11), 1410-1415.

Mehdizadeh, M., Lajevardi, L., Habibi, S. A. H., ArabBaniasad, M., Baghoori, D., Daneshjoo, F., \& Taghiza- 
deh, G. (2016). The association between fear of falling and quality of life for balance impairments based on hip and ankle strategies in the drug On-and Off-phase of patients with idiopathic Parkinson'disease. Medical journal of the Islamic Republic of Iran, 30, 453.

Mehdizadeh, M., Mehraban, A. H., \& Zahediyannasab, R. (2017). The effect of group-based occupational therapy on per-formance and satisfaction of stroke survivors: Pilot trail, neuro-occupational view. Basic and clinical neuroscience, 8(1), 69.

Monfared, N. R. S., Mehraban, A. H., \& Boroumand, S. (2015). Effectiveness of Videogames on Balance and Fear of Falling in Chronic Stroke Patient. Iranian Rehabilitation Journal, 13(1).

Mozaffarian, D., Benjamin, E., Go, A., Arnett, D. K., Blaha, M. J., Cushman, M., Fullerton, H. J. (2015). Aha statistical update. Heart disease and stroke.

Rosén, E., Sunnerhagen, K. S., \& Kreuter, M. (2005). Fear of falling, balance, and gait velocity in patients with stroke. Physio-therapy theory and practice, 21(2), 113120.

Salavati, M., Negahban, H., Mazaheri, M., Soleimanifar, M., Hadadi, M., Sefiddashti, L., Feizi, A. (2012). The Persian version of the Berg Balance Scale: inter and intra-rater reliability and construct validity in elderly adults. Disability and rehabilitation, 34(20), 1695-1698. Schinkel-Ivy, A., Inness, E. L., \& Mansfield, A. (2016). Relationships between fear of falling, balance confidence, and control of balance, gait, and reactive stepping in individuals with sub-acute stroke. Gait \& posture, 43 , 154-159.

Schmid, A. A., Arnold, S. E., Jones, V. A., Ritter, M. J., Sapp, S. A., \& Van Puymbroeck, M. (2015). Fear of falling in people with chronic stroke. American Journal of Occupational Therapy, 69(3).

Shaikh, M., \& asghar Hosseini, H. (2016). Fear of Falling in Patients with Chronic Stroke: Differences of Functional Gait and Balance Measures According to the Level of Concern about Falling. Journal of Rehabilitation Sciences and Research, 3(2), 35-38.

Smith, P. S., Hembree, J. A., \& Thompson, M. E. (2004). Berg Balance Scale and Functional Reach: determining the best clini-cal tool for individuals post acute stroke. Clinical rehabilitation, 18(7), 811-818.

Tinetti, M. E., Williams, T. F., \& Mayewski, R. (1986). Fall risk index for elderly patients based on number of chronic disabili-ties. The American journal of medicine, 80(3), 429-434. 
Function and Disability Journal ISSN: 2588-6304

$$
\text { مقالة يزوهشى }
$$

\section{استفاده از ابزارهاى سنجش تعادل براى ييشبينى ترس از افتادن در افراد مبتلا به سكتُ}

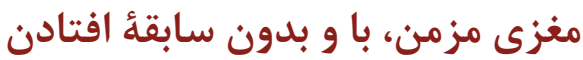

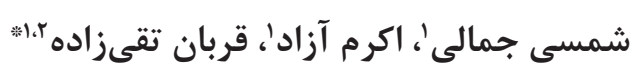

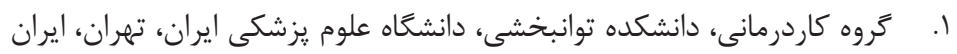

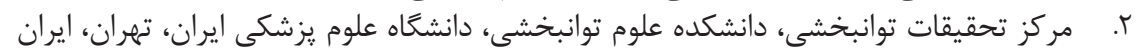

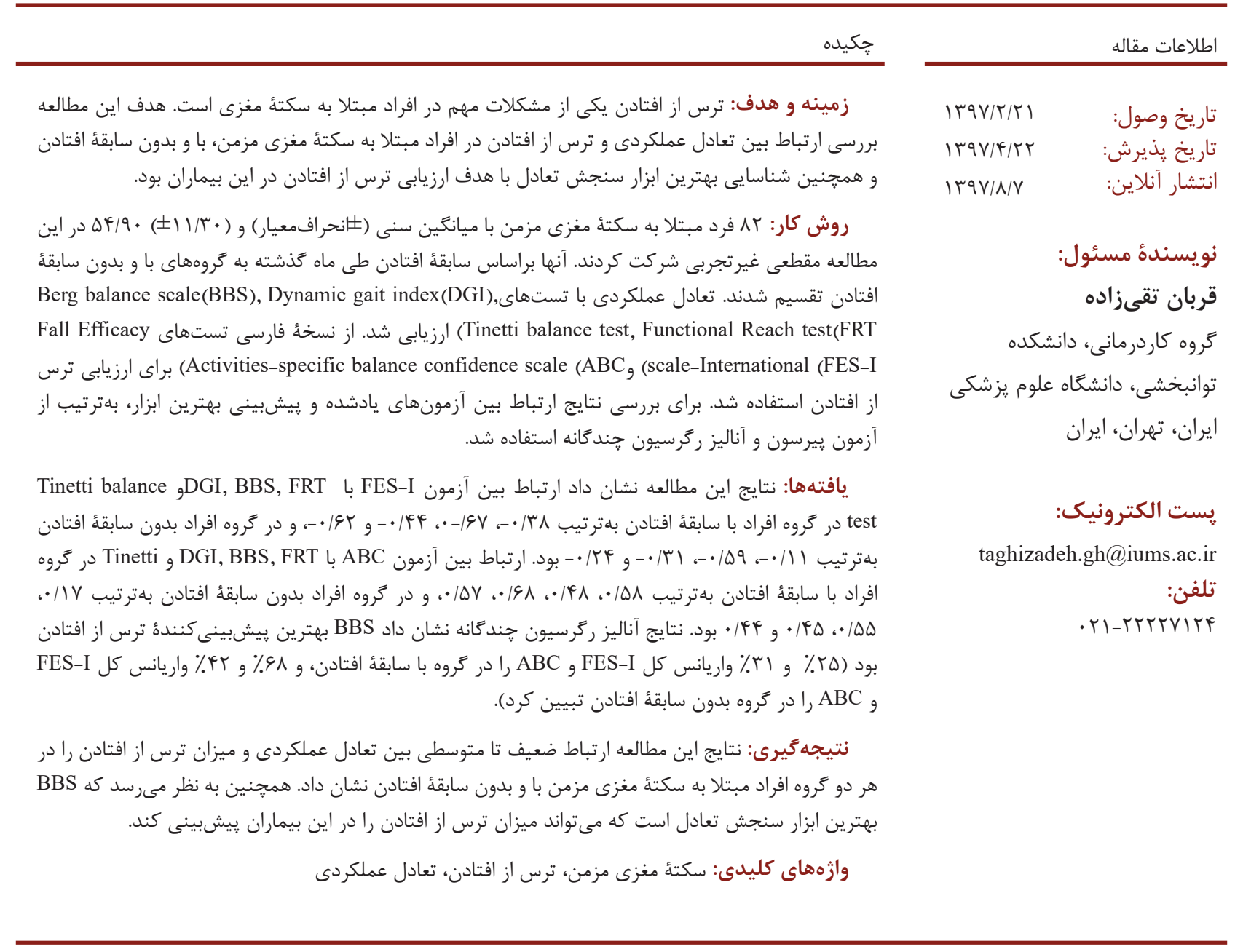


Ghorban Taghizadeh et al .28 